\title{
Asymmetric Information Supply Chain Models with Credit Option
}

\author{
Xu Zhang, Panlop Zeephongsekul* \\ School of Mathematical and Geospatial Sciences, RMIT University, Melbourne, VIC, Australia
}

(Received: January 15, 2013 / Revised: April 11, 2013 / Accepted: August 18, 2013)

\begin{abstract}
Credit option is a policy that has been studied by many researchers in the area of supply chain management. This policy has been applied in practice to improve the profits of supply chain members. Usually, a credit option policy is proposed by the seller, and often under a symmetric information environment where members have complete information on each others' operations. In this paper, we investigate two scenarios: firstly, the seller offers a credit option to the buyer, and secondly, the buyer attempts to stretch the length of the credit period offered by the seller. The proposed model in both scenarios will be investigated under an asymmetric information structure where some information are private and are only known to the individual who has knowledge of this information. The interactions between buyer and seller will be modeled by non-cooperative Stackelberg games where the buyer and seller take turn as leader and follower. Among some of the numerical results obtained, the seller and buyer's profits obtained from symmetric information games are larger than those obtained from an asymmetric information game in both scenarios. Furthermore, both buyer and seller's profit in the second scenario are better than in the first scenario.
\end{abstract}

Keywords: Seller-Buyer Supply Chain, Credit Option, Asymmetric Information Pattern, Stackelberg Game

* Corresponding Author, E-mail: panlop.zeephongsekul@rmit.edu.au

\section{INTRODUCTION AND LITERATURE REVIEW}

During the last few decades, numerous research papers have appeared in the area of supply chain management under various assumptions and from different perspective of the participants in the chain, and by applying different methodologies. One of the main objectives of these papers is to obtain supply chain policies which will improve coordination between the various channels in the supply chain, as well as achieving the best outcomes efficiently and effectively among the channel members. One of the means of achieving this objective is to implement a trade credit policy and this has attracted many researchers' attention since Haley and Higgins (1973) studied the buyer's lot-sizing problem under a trade credit contract.
They showed that under the assumption of a constant demand, the lot size of the buyer is invariant with respect to the length of the credit period. Subsequently, other studies have appeared in the literatures which are related to this problem. Goyal (1985) considered the buyer's problem under trade credit agreement based on the assumption that interest rate for credit balance and credit deficit are different. Kim et al. (1995) proposed a model from the seller's perspective to determine the optimal credit period under the assumption that the seller's selling price is fixed. Shinn (1997) showed that if demand is price sensitive, then the order quantity of the buyer is not invariant with respect to the length of the credit period. Related to this, Aggarwal and Jaggi (1995), Chung (1998), and Jamal et al. (2000) studied the deteriorating item problem with permissible delay in payment under the assumption that demand is 
constant. Jaber and Osman (2006) studied a two-level supply chain model where the players coordinate their orders in order to minimize local cost. They proposed a policy in which the seller uses trade credit as an incentive to increase the order quantity of the buyer, and will charge interest if the buyer's payment is overdue. Since the demand in their paper is assumed constant, the credit period offered by the seller will not affect the result of the transaction. We also refer to Thangam and Uthayakumar (2009), Ouyang et al. (2009), Chen and Kang (2010), and Zhong and Zhou (2012) for more recent papers which are related to the work reported in this paper.

All the aforementioned papers studied the credit period with permissible delay in payment policy purely from an inventory management viewpoint, totally ignoring the interaction between players and the consequences of this interaction. In recent years, the game theory has emerged as an essential tool in investigating the interaction between players in a seller-buyer supply chain. Esmaeili et al. (2009) introduced several game - theoretic supply chain models based on symmetric information game, and these were later extended to an asymmetric information scenario in Esmaeili and Zeephongsekul (2010). For work related to trade credit option, we should also mention Abad and Jaggi (2003) who proposed a joint approach of seller-buyer channel to determine the unit price and length of credit period for the seller using a game theoretic approach. They investigated a leader-follower relationship based on Stackelberg game where seller is the leader and the buyer the follower. They also present a Nash bargaining solution with the Stackelberg game solution as a fixed threat. In their paper, the rate of the opportunity cost of capital for the seller is assumed linear to the credit period, and the buyer will settle the payment immediately after the credit period. Similarly, in a recent work by Zhou et al. (2012), the authors studied two-echelon supply chain by implementing Stackelberg game to design a trade credit policy for the supplier to achieve optimal annual profit. However, in both of these papers, the models are based on the assumption that the information about certain parameters in the supply chain, such as demand and interest rate, are common knowledge to both players, i.e., they are symmetric. However, in reality, these information are private to each individual player who may not be willing to reveal them to the other player.

In this paper, we investigate two scenarios regarding credit period policy by extending the model in Abad and Jaggi (2003) to the asymmetric information case. In the first scenario, the seller offers a credit period to the buyer as an incentive strategy to entice the latter to increase the order quantity. During the credit period, the seller will carry the inventory cost for the buyer, while the latter can enjoy capital gain. The seller's capital cost rate is assumed to be linearly related to the length of the credit period as in Abad and Jaggi (2003). We assume that demand is sensitive to both selling price and marketing expenditure, and known to the buyer, while the seller is unaware of this demand. The seller will determine the optimum length of credit period based on the uncertainty of the demand. It is clear that in this scenario, if the buyer does not settle the payment immediately after the credit period, the seller will end up losing capital. In the second scenario,

Table 1. Decision variables and parameters

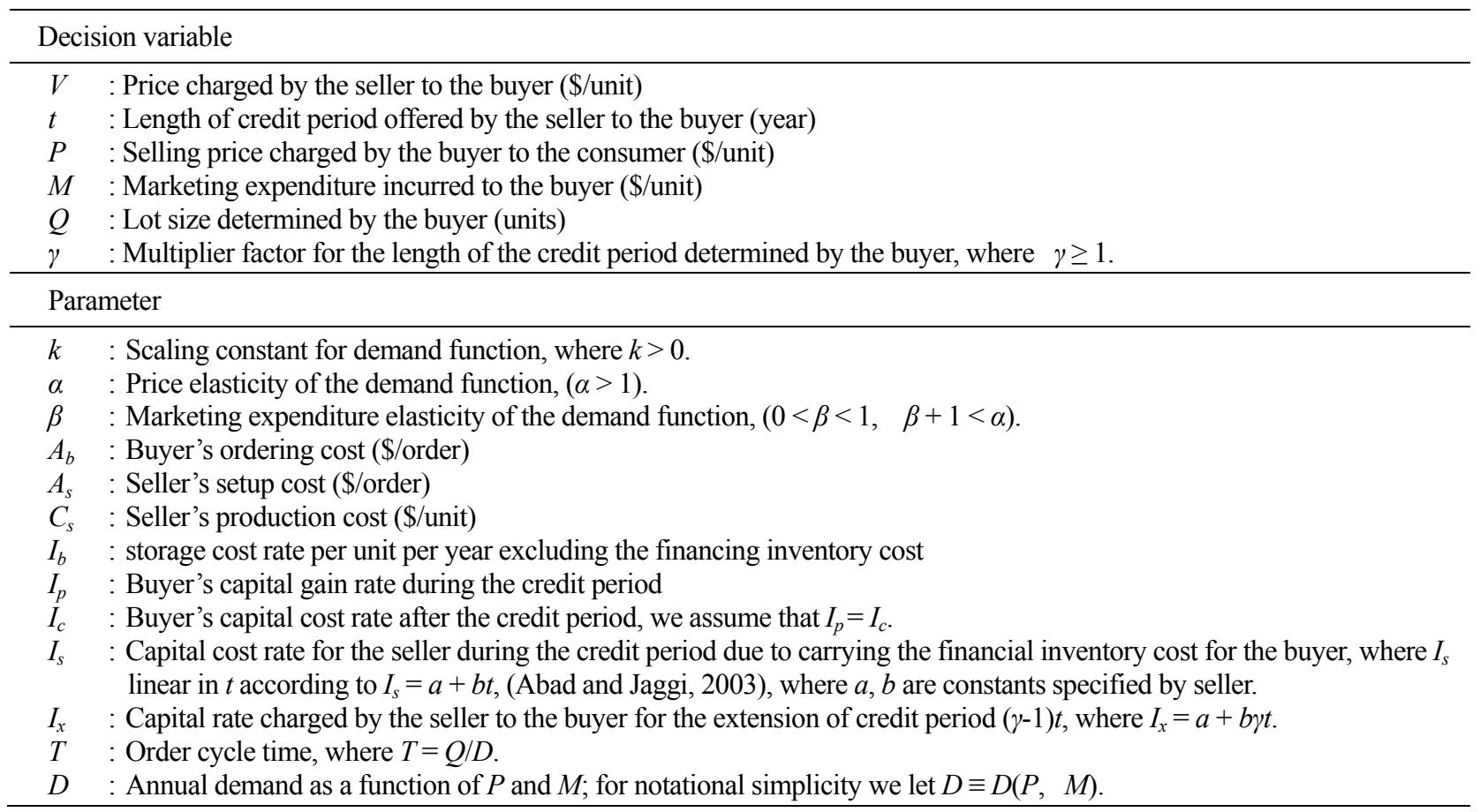


the buyer seeks to extend the length of the credit period by offering to share the inventory cost incurred by the seller. The buyer would like to extend the length of the credit period by a certain multiplier factor with an offer of accepting charges from the seller with interest rate linearly related to the extension period. All relevant information concerning the interest rate is only known to the seller, but the buyer determines the multiplier factor based on her uncertainty concerning this interest rate. We will investigate the interaction between seller and buyer as a non-cooperative Stackelberg game, where each player takes turns as leader and follower. One of the main reasons for applying Stackelberg game to our supply chain model is due to an observation prevailing in the market place where in some situations some players have more power than others. So the strategies of each player would depend on their leadership position in the game. Numerical results show that both seller and buyer's profits obtained from symmetric information game are larger than those obtained from asymmetric information game; moreover, the profit for both seller and buyer in the second scenario are better than those obtained in the first scenario. We also examined the player's belief on other player's type using different probability density functions (PDF). The results show that players' decisions are sensitive to their choice of PDF.

After this introduction, the notation, assumptions and model formulations, from both the seller's and buyer's perspectives, are provided in Section 2. In Section 3, we present the Seller-Stackelberg game, where the seller is the leader and determines the optimum length of the credit period. In Section 4, we investigate the BuyerStackelberg game, where the buyer is the leader and determines the optimum multiplier factor of the extended length of credit period. In Section 5, we provide numerical examples to compare the different models and discuss the effects that incomplete information has on the players' decisions and profits. We also provide a sensitivity analysis on players' decisions based on different PDFs assumed by them. Finally, the paper is concluded in Section 6 with some suggestions for future work. To improve the readability, we will refer seller with the masculine $h e$ and the buyer with the feminine she.

\section{NOTATION, ASSUMPTION AND MODEL FORMULATION}

The following notation and assumptions will be used in our models, which comprise the profit functions from both the perspectives of the buyer and seller.

\subsection{Notation and Assumption}

The decision variables and parameters are presented in Table 1 and proposed models are based on the following assumptions:

1) Planning horizon is infinite.

2) The annual demand is sensitive to both selling price and marketing expenditure according to (Lee and Kim, 1993):

$$
D=k P^{-\alpha} M^{\beta}
$$

3) Shortages are not permitted.

4) The buyer has knowledge of the values of $\alpha$ and $\beta$ and therefore is aware of the demand function. Ho wever, she is unaware of the parameters $a$ and $b \mathrm{w}$ hich are known to the seller. All other parameter $\mathrm{s}$ are common knowledge to both players. This is therefore a two-person game with asymmetric info rmation (Rasmusen, 2007).

\subsection{Model Formulation}

We will formulate a general model for both buyer and seller based on the second scenario described in Section 1, the model used in first scenario is a special case of this general model.

\subsubsection{The buyer's model}

In the second scenario, in order to ease financial pressure such as cash flow problem, the buyer would like to extend the length of credit period and in return offers to share the seller's inventory cost at an interest rate of $I_{x}$. This extension could also improve the profit of both the buyer and the seller. The buyer's problem is to determine the selling price, marketing expenditure, order quantity and multiplier factor $\gamma$ such that her net profit will be maximized. With capital gain (Abad and Jaggi, 2003) in the credit period, the buyer's profit is calculated as:

\section{Buyer's profit $=$ Sales revenue - Purchase cost - Marketing cost-Ordering cost + Capital gain due credit period - Storage cost excluding financing cost - Inventory financing cost for the extended credit period.}

There are three cases for the buyer's model which are described in Figure 1 below.

The shaded areas refer to the capital gain for the buyer and $[0, t]$ is the credit period offered by the seller where the buyer would like to extend to $[0, \gamma t]$.

In case 1 , the buyer will have a capital gain during the extended credit period of $0.5 I_{p} V D(\gamma t)^{2}$, and the inventory cost for the same period is $0.5 I_{c} V D(T-\gamma t)^{2}$.

In cases 2 and 3 , the capital gain during the extended credit period is $0.5 I_{p} V D T^{2}+I_{p} V D T(\gamma t-T)$, but there is no inventory cost for both cases.

In all three cases, the compensation for seller's in- 

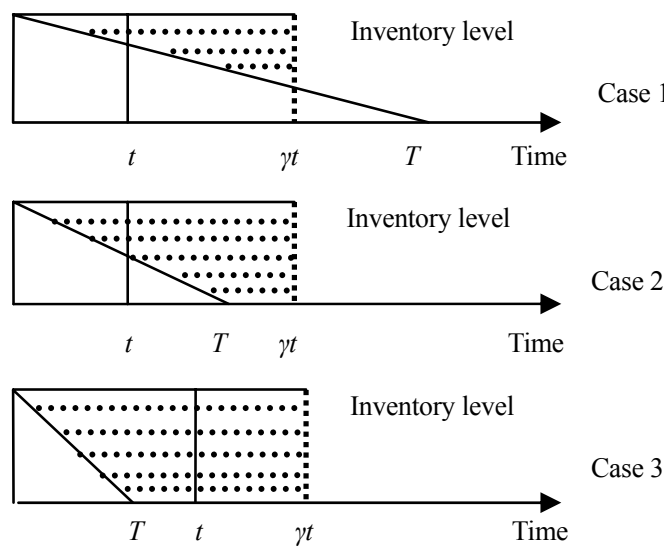

Figure 1. Time span for buyer's model.

ventory cost put up by the buyer during the extended credit period is $I_{x} V D T(\gamma t-t)$. We refer the reader to the Appendix for a proof that using assumptions $I_{c}=I_{p}$ and $T=Q / D$, it can be shown that the buyer's profit function in all three cases have the same mathematical expression. Therefore, the buyer's annul profit function is:

$$
\begin{aligned}
\Pi_{b}(P, M, Q, \gamma) & =P D-V D-M D-A_{b} D Q^{-1}-0.5 I_{b} V Q \\
& +I_{p} V D \gamma t-0.5 I_{p} V Q-I_{x} V D(\gamma t-t) \\
& =D\left(P-V\left(1+\left(I_{x}-I_{p}\right) \gamma t-I_{x} t\right)-M-A_{b} Q^{-1}\right] \\
& -0.5 V Q\left(I_{b}+I_{p}\right)
\end{aligned}
$$

It can be shown that the buyer's profit function is strictly Pseudoconcave in $P$-c.f. to Esmaeili et al. (2009), and it is also concave in $M, Q$, and $\gamma$. The first order condition with respect to $\gamma, P, M$, and $Q$, yield the following equations:

$$
\begin{gathered}
\gamma=\frac{b t-a+I_{p}}{2 b t} \\
P=\frac{\alpha\left\{\left(V+A_{b} Q^{-1}\right)+V t\left[(\gamma-1)(a+b t \gamma)-I_{p} \gamma\right]\right\}}{\alpha-\beta-1} \\
M=\frac{\beta\left\{\left(V+A_{b} Q^{-1}\right)+V t\left[(\gamma-1)(a+b t \gamma)-I_{p} \gamma\right]\right\}}{\alpha-\beta-1} \\
Q^{2}\left[\left(V+A_{b} Q^{-1}\right)+V t\left((\gamma-1)(a+b t \gamma)-I_{p} \gamma\right)\right]^{\alpha-\beta} \\
=\frac{2 k A_{b} \beta^{\beta}(\alpha-\beta-1)^{\alpha-\beta}}{\alpha^{\alpha} V\left(I_{b}+I_{c}\right)}
\end{gathered}
$$

For a given $V$ and $t$, the buyer's optimal decision variables can be obtained by solving the above equations simultaneously.

\subsubsection{The seller's model}

The seller's objective is to determine the selling price and the length of credit period such that his profit will be maximized. The seller's profit function can be expressed as:

Seller's profit $=$ Sales revenue - Production cost - Setup cost Capital cost in credit period + Capital gain during extended credit period.

According to the assumptions, the seller will incur the capital rate $I_{S}$ for the cost of financially carrying inventory for the buyer during the credit period, and impose the rate $I_{x}$ for extending the credit period. Hence, the capital cost during period $[0, t]$ is $I_{s} V D t$, and the capital gain in period $[t, \gamma t]$ is $I_{x} V D(\gamma t-t)$ and the seller's profit can be expressed mathematically as:

$\Pi_{s}(V, t)=V D-C_{s} D-A_{s} D Q^{-1}-I_{s} V D \gamma t+I_{x} V D(\gamma t-t)$

Since $\frac{\partial^{2} \Pi_{s}}{\partial t^{2}}<0$, the first order condition with respect to $t$, yields:

$$
t^{*}=\frac{a}{2 b \gamma(\gamma-2)}
$$

Notice that $\Pi_{\mathrm{s}}(V, t)$ is linear in $V$, so the seller's selling price is unbounded. It is reasonable for seller to set the selling price as:

$$
V^{*}=F V_{0}=\frac{4 F b \gamma\left(C_{s}+A_{s} Q^{-1}\right)(\gamma-2)}{4 b \gamma(\gamma-2)-a^{2}}
$$

where

$$
V_{0}=\frac{4 b \gamma\left(C_{s}+A_{s} Q^{-1}\right)(\gamma-2)}{4 b \gamma(\gamma-2)-a^{2}}
$$

is the price when there is no profit. Note that $F>1$ can be obtained through negotiation with the buyer.

\section{CREDIT PERIOD: THE FIRST SCENARIO}

In this scenario, the seller offers a credit period to the buyer with the aim of inducing the buyer to increase her order quantity. We assume here that the demand is unknown to the seller, i.e., he has no knowledge of $\alpha$ and $\beta$ in the demand function. The seller's problem is to determine the selling price $V$ and the length of credit period $t$ such that his profit will be maximized. Adhering to the classical approach in games with incomplete information pioneered in Harsanyi (1967, 1968a, 1968b), we identify the buyer's type space as $R=\{\alpha, \beta\}$ where $\alpha>\beta+1$ and $0<\beta<1$ and denote a typical member of $R$ by $r$. We let the PDF $f_{R}(r)$ denotes the seller's uncertainty over the buyer's type. We will present a Seller-Stackelberg game, i.e., seller is the leader and buyer the follower, under an asymmetric information structure. We also give solution 
for the game under a symmetric information structure, i.e., when there is no uncertainty regarding the demand.

\subsection{Asymmetric Seller-Stackelberg Game}

Under this scenario, the buyer's profit function is obtained by setting $\gamma=1$ in Eq. (2), resulting in

$$
\begin{aligned}
\Pi_{b}(P, M, Q) & =P D-V D-M D-A_{b} D Q^{-1} \\
& +I_{p} V D t-0.5 I_{b} V Q-0.5 I_{c} V Q
\end{aligned}
$$

and the seller's profit function is

$$
\Pi_{s}(V, t)=V D-C_{s} D-A_{s} D Q^{-1}-I_{s} V D t .
$$

Which is obtained from (7) by setting $\gamma=1$.

The seller's objective, when he is the leader, is to seek the optimal $V$ and $t$ based on the buyer's optimal decision variables which are given by Eqs. (4)-(6) when $\gamma$ $=1$. Since the seller is uncertain of the buyer's type, he will seek to maximize his expected profit with respect to the PDF $f_{R}(r)$ over the buyer's type space. Hence, the seller's problem becomes:

$$
\begin{aligned}
& \operatorname{Max}_{r}\left(\Pi_{s}(V, t)\right) \\
& =\int_{R}\left(V D-C_{s} D-A_{s} D Q^{-1}-I_{s} V D t\right) f_{R}(r) d r \\
& \text { Subject to } P=\frac{\alpha\left(V+A_{b} Q^{-1}-I_{p} V t\right)}{\alpha-\beta-1} \\
& M=\frac{\beta\left(V+A_{b} Q^{-1}-I_{p} V t\right)}{\alpha-\beta-1} \\
& Q^{2}\left(V+A_{b} Q^{-1}-I_{p} V t\right)^{\alpha-\beta}=\frac{2 k A_{b} \beta^{\beta}(\alpha-\beta-1)^{\alpha-\beta}}{\alpha^{\alpha} V\left(I_{b}+I_{c}\right)}
\end{aligned}
$$

Substituting $P$ and $M$ given by (13) and (14), respectively into the objective function (12), reduce the problem to:

$$
\begin{aligned}
\operatorname{Max}_{r}\left(\Pi_{s}(V, t)\right) \\
=\int_{R}\left[k \alpha^{-\alpha} \beta^{\beta}(\alpha-\beta-1)^{\alpha-\beta}\left(V+A_{b} Q^{-1}-I_{p} V t\right)^{-\alpha+\beta}\right. \\
\left.\quad\left(V-C_{s}-A_{s} Q^{-1}-I_{s} V t\right)\right] f_{R}(r) d r
\end{aligned}
$$

with Eq. (15) as constraint. Using any nonlinear programming search tool, it is straightforward to obtain the optimal solution once $f_{R}(r)$ is given. A numerical example will be presented in Section 5 .

\subsection{Symmetric Seller-Stackelberg Game}

Under symmetric information, both seller and buyer's information regarding to their operations are common knowledge. Therefore, the element of randomness can be ignored and we can omit the expectation operator in Eq. (16), expressing seller's problem for a symmetric SellerStackelberg game as:

$$
\begin{aligned}
& \operatorname{Max}_{s}(V, t)=k \alpha^{-\alpha} \beta^{\beta}(\alpha-\beta-1)^{\alpha-\beta} \\
& \quad\left(V+A_{b} Q^{-1}-I_{p} V t\right)^{-\alpha+\beta}\left(V-C_{s}-A_{s} Q^{-1}-I_{s} V t\right)
\end{aligned}
$$

$$
\begin{aligned}
\text { Subject to } & \left(V+A_{b} Q^{-1}-I_{p} V t\right)^{\alpha-\beta} Q^{2} \\
= & \frac{2 k A_{b} \beta^{\beta}(\alpha-\beta-1)^{\alpha-\beta}}{\alpha^{\alpha} V\left(I_{b}+I_{c}\right)}
\end{aligned}
$$

To solve this problem, we obtain the largest positive root of Eq. (18), then substituting this into objective function (17), reducing the problem into a non-constrained nonlinear optimization problem. We can then solve this problem numerically using any nonlinear optimization search tool.

\section{CREDIT PERIOD: THE SECOND SCENARIO}

In the second scenario, the buyer seeks to extend her credit period in order to alleviate her financial pressure and improve profit. The credit period will be extended by a factor $\gamma$, i.e., the length of credit period will be extended by $(\gamma-1) t$. In return, the buyer will reimburse the seller for this extension by allowing the seller to charge her an interest rate $I_{x}$ on the inventory cost that is incurred. In this scenario, the buyer becomes the leader since she determines the factor $\gamma$. We will investigate the BuyerStackelberg game, i.e., when she is leader and seller is the follower, under a symmetric as well as an asymmetric information structure. The buyer's objective is to determine the $\gamma$, selling price $P$, marketing expenditure $M$, and order quantity $Q$, based on the seller's best strategy which would maximize his profit.

\subsection{Symmetric Buyer-Stackelberg Game}

Suppose information is symmetric, the buyer will maximize her profit based on seller's best strategy $V$ and $t$ which is given by Eqs. (8) and (9). The buyer's problem becomes:

$$
\begin{aligned}
\operatorname{Max} & \Pi_{b}(P, M Q \gamma) \\
= & D\left[P-V\left(1+\left(I_{x}-I_{p}\right) \gamma t-I_{x} t\right)-M-A_{b} Q^{-1}\right] \\
& -0.5 V Q\left(I_{b}+I_{p}\right)
\end{aligned}
$$

Subject to $V=\frac{4 F b \gamma\left(C_{s}+A_{s} Q^{-1}\right)(\gamma-2)}{4 b \gamma(\gamma-2)-a^{2}}$ 


$$
\begin{gathered}
t=\frac{a}{2 b \gamma(\gamma-2)} \\
\gamma>2
\end{gathered}
$$

To solve this problem, we substituting Eqs. (20) and (21) into (19), and the problem reduces to an nonlinear optimization problem with constraint (22). The numerical solutions for $P^{*}, M^{*}, Q^{*}$, and $\gamma^{*}$ can be obtained by using a nonlinear optimization routine.

\subsection{Asymmetric Buyer-Stackelberg Game}

Suppose the buyer is unaware of $a$ and $b$, which are the parameters associated with the interest rate $I_{x}$, and the buyer will have to determine her optimal strategy based on this uncertainty. The seller's type space is $S=\{a, b\}$, where $a>0$ and $b>0$. We denote a typical member of $S$ by $s$. We let the PDF $f_{S}(s)$ denotes the buyer's uncertainty over the seller's type. The buyer seeks optimal $P, M, Q$, and $\gamma$ based on the seller's best decision variables, which are given by Eq. (8) and (9). Since the buyer is not aware of the seller's type, she will maximize her expected profit with respect to $\operatorname{PDF} f_{S}(s)$, and the buyer's problem becomes:

$$
\begin{aligned}
& \operatorname{Max}_{s}\left(\Pi_{b}(P, M, Q, \gamma)\right) \\
& =\int_{S} D\left[P-V\left(1+\left(I_{x}-I_{p}\right) \gamma t-I_{x} t\right)-M-A_{b} Q^{-1}\right] f_{S}(s) d s \\
& -\int_{S}\left[0.5 V Q\left(I_{b}+I_{p}\right)\right] f_{S}(s) d s \\
& \text { subject to } V=\frac{4 F b \gamma\left(C_{s}+A_{s} Q^{-1}\right)(\gamma-2)}{4 b \gamma(\gamma-2)-a^{2}} \\
& t=\frac{a}{2 b \gamma(\gamma-2)} \\
& \gamma>2
\end{aligned}
$$

Once $f_{S}(s)$ is given, substituting Eqs. (24) and (25) into the buyer's objective function (23) will result in a nonlinear programming problem constrained by Eq. (26).

\section{NUMERICAL RESULTS}

In this section, we will present several numerical examples to illustrate the proposed models. In the examples, we will assume that the following parameters are common knowledge to both players, i.e., $k=36,000, F=1.8$, $L=0.088, A_{b}=38, A_{s}=140, C_{s}=1.5, I_{b}=0.1, I_{c}=I_{p}=$ 0.15 . In the symmetric game we let $\alpha=1.7, \beta=0.15, a=$ 0.08 , and $b=0.05$. In the Seller-Stackelberg asymmetric game, in order to facilitate our presentation, we will follow Sethuraman and Tellis (1991) by letting $\alpha$ and $\beta$ be related by $\beta=L \alpha$. We also assume that $\alpha$ is uniformly distributed with mean $\bar{a}=1.7$ and standard deviation $\sigma_{\alpha}=0.1$. In the Buyer-Stackelberg asymmetric game, we assume that parameters $a$ and $b$ are independent and uniformly distributed with mean $\bar{a}=0.08, \bar{b}=0.05$ and corresponding standard deviation $\sigma_{a}=0.05, \sigma_{b}=0.05$. Based on the numerical results, the effect of asymmetric information on players' decisions and profits will be discussed as well as the results obtained from the two scenarios. In the following examples, the first two deal with the first scenario and the last two with the second scenario.

\subsection{Numerical Examples}

Example 1. This example deals with the Seller-Stackelberg symmetric game. Solving problem (17) constrained by Eq. (18), we obtain the seller's optimal profit as $\Pi_{s}^{*}=862.70$ with optimal selling price $V^{*}=4.85$ and the length of credit period $t^{*}=0.5520$. The buyer's optimal selling price $P^{*}=$ 14.48 , marketing expenditure $M^{*}=1.27$ and order quantity $Q^{*}=157.77$, yielding the buyer an optimal profit $\Pi_{b}^{*}=$ 3285.63 using Eq. (10).

Example 2. In the Seller-Stackelberg asymmetric game, by incorporating the given PDF $f_{R}(r)$ into Eq. (16) and solving the resulting problem constrained by Eq. (15), we obtained the seller's expected profit as $E_{r}\left(\Pi_{s}^{*}\right)=858.63$ with optimal selling price $V^{*}=4.89$ and length of credit period $t^{*}=0.5516$. The corresponding buyer's optimal profit is $\Pi_{b}^{*}=3270.13$ obtained using Eq. (10); Eqs. (13) to $(15)$ give $P^{*}=14.60, M^{*}=1.28$, and $Q^{*}=156.09$.

Example 3. In the Buyer-Stackelberg symmetric game using the second scenario, the buyer takes the lead by offering to extend the credit period and the seller acquiesce. Solving problem (19) constrained by Eqs. (20) to (22), we obtain the buyer's optimal profit as $\Pi_{b}^{*}=$ 4116.23 with optimal selling price $P^{*}=8.86$, marketing expenditure $M^{*}=0.78$, order quantity $Q^{*}=821.05$, and multiplier factor $\gamma=2.51$. Also, from Eqs. (8) and (9), we obtain the seller's optimal selling price $V^{*}=3.08$ and the length of credit period $t^{*}=0.6247$, resulting in the seller's optimal profit of $\Pi_{s}^{*}=1136.94$ using Eq. (7).

Example 4. For the Buyer-Stackelberg asymmetric game, incorporating $\operatorname{PDF} f_{S}(s)$ into Eq. (23), and solving this problem under the constraints (24) to (26), we obtain the buyer's optimal selling price as $P^{*}=9.29$, marketing expenditure $M^{*}=0.82$, order quantity $Q^{*}=812.76$, and multiplier factor $\gamma=3.20$ giving the buyer's expected profit $E_{s}\left(\Pi_{b}^{*}\right)=4064.69$. The corresponding seller's profit $\Pi_{s}^{*}=$ 1056.67 can be obtained from Eq. (7) using $V^{*}=3.04$ and $t^{*}$ $=0.2091$ obtained from Eqs. (8) and (9), respectively. 


\subsection{Discussions}

Comparing the results obtained from symmetric and asymmetric information game in the first scenario, the seller's selling price in the first game is less than the second game, but with a longer credit period; on the other hand, the buyer's order quantity in the symmetric game is larger than the asymmetric game, but the selling price and marketing expenditure are less. Thus, both seller and buyer's profit in the symmetric game are larger than in the asymmetric game. The same results prevail in the second scenario, although the multiplier factor $\gamma$ in the asymmetric game is larger than in the symmetric game. These observations tend to lend credence to the fact that asymmetric information will affect both seller and buyer's decisions, resulting in a less profitable solution as a consequence.

Figures 2 and 3 below highlight the solutions for both seller and buyer obtained from symmetric game versus those obtained from asymmetric game, for both Seller- and Buyer-Stackelberg game, respectively.

We next compare the results obtained from the two scenarios. Comparing the Buyer-Stackelberg symmetric game in the second scenario with the Seller-Stackelberg asymmetric game in the first scenario indicates that the buyer's profit increased by $25.87 \%$ and the seller by $32.41 \%$. Comparison with the Seller-Stackelberg symmetric game indicates that the buyer's profit increased by $25.28 \%$, and the seller's profit by $31.79 \%$. Comparison of the Buyer-Stackelberg asymmetric game in the second scenario with the Seller-Stackelberg asymmetric game in the first scenario indicates an increase in profit of $24.3 \%$

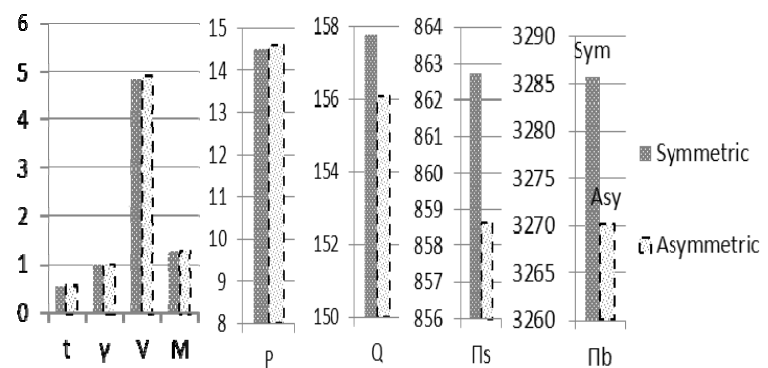

Figure 2. First scenario of symmetric vs. asymmetric game.

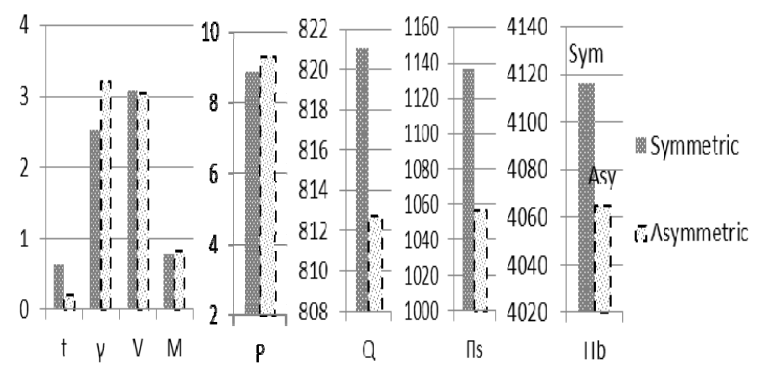

Figure 3. Second scenario of symmetric vs. asymmetric game.

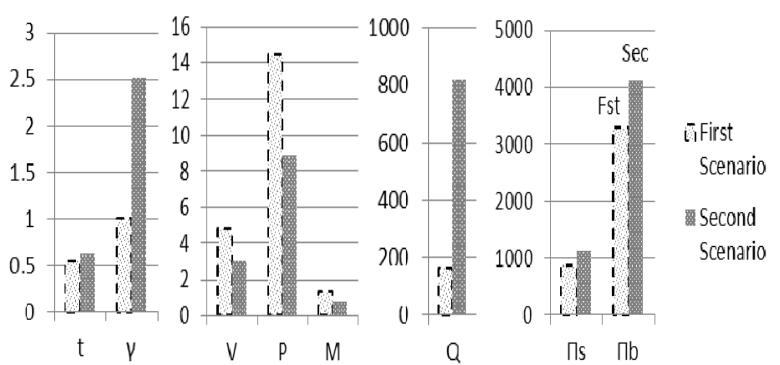

Figure 4. Symmetric game of first vs. second scenario.

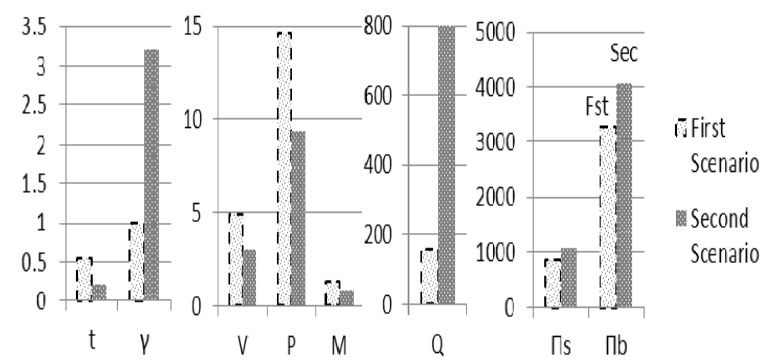

Figure 5. Asymmetric game of first vs. second scenario.

for the buyer and $23.06 \%$ for the seller. Comparison with the Seller-Stackelberg symmetric game shows an increase in profit of $23.71 \%$ for the buyer and $22.48 \%$ for the seller. Therefore, irrespective of the information status of the buyer or seller, their profits have improved in the second scenario. This points to the benefit of extending the length of the credit period by the buyer irrespective of the information status of the players.

Figures 4 and 5, highlight solutions obtained from the first scenario versus those obtained from the second scenario for both symmetric game and asymmetric game.

\subsection{Sensitivity Analysis Based on Choice of PDF}

Under asymmetric information, a player's belief or uncertainty on other player's type may affect their decisions and profit through his choice of PFD. In order to gain a better understanding of the effect on the outcomes of the game based on their choice of PDF, we provide the following sensitivity analysis. The chosen PDFs are the uniform, exponential, and normal PDFs. In the first scenario, Seller-Stackelberg game, we assume that buyer's type $\alpha$ has mean $\bar{a}=1.7$ and standard deviation $\sigma_{a}=0.1$ for all three distributions; Similarly, in the second scenario, Buyer-Stackelberg game, with respect to seller's type $a$ and $b$, in the chosen distributions, $a$ and $b$ are assumed independent with mean $\bar{a}=0.08, \bar{b}=0.05$ and corresponding standard deviation $\sigma_{a}=0.05, \sigma_{b}=0.05$. All other parameters remain unchanged from that given in the preceding examples. The numerical results for both SellerStackelberg game (first scenario) and Buyer-Stackelberg 
Table 2. Results of first scenario with respect to probability density function

\begin{tabular}{crrr}
\hline & Uniform & Exponential & Normal \\
\hline $\mathrm{t}$ & 0.5516 & 0.5513 & 0.552 \\
$\gamma$ & 1 & 1 & 1 \\
$\mathrm{~V}$ & 4.89 & 4.91 & 4.85 \\
$\mathrm{P}$ & 14.6 & 14.68 & 14.48 \\
$\mathrm{M}$ & 1.28 & 1.29 & 1.27 \\
$\mathrm{Q}$ & 156.09 & 155.05 & 157.77 \\
$\Pi_{\mathrm{s}}$ & 858.63 & 862.62 & 862.7 \\
$\Pi_{\mathrm{b}}$ & 3270.13 & 3260.62 & 3285.58 \\
\hline
\end{tabular}

Table 3. Results of second scenario with respect to probability density function

\begin{tabular}{rrrr}
\hline & Uniform & Exponential & Normal \\
\hline $\mathrm{t}$ & 0.2092 & 0.6323 & 0.523 \\
$\gamma$ & 3.2 & 2.51 & 2.59 \\
$\mathrm{~V}$ & 3.04 & 3.08 & 3.07 \\
$\mathrm{M}$ & 0.82 & 0.81 & 0.8 \\
$\mathrm{P}$ & 9.29 & 9.22 & 9.04 \\
$\mathrm{Q}$ & 812.76 & 822.82 & 817.75 \\
$\Pi_{\mathrm{b}}$ & 4064.69 & 4113.22 & 4112.71 \\
$\Pi_{\mathrm{s}}$ & 1056.67 & 1066.56 & 1102.43 \\
\hline
\end{tabular}

game (second scenario) regarding different PDF are summarized in the following table.

From Table 2, with respect to buyer's type $\alpha$, when seller beliefs that $\alpha$ is normally distributed, the seller's selling price $V$ will be smaller and the length of credit period $t$ will be larger compared with those from the uniform or exponential distribution. Also, buyer's selling price $P$ and marketing effort $M$ are smaller, but order quantity $Q$ is larger. Therefore, a larger profit for both seller and buyer will result if buyer's type $\alpha$ is normally distributed rather than uniform or exponentially distributed.

Table 3 shows that when a buyer believes that $a$ and $b$ are exponentially distributed, the buyer will order a larger quantity $Q$ with smaller multiplier factor $\gamma$, and the seller will set a larger selling price $V$ and a longer length of credit period $t$ when compare with the other two distributions. If $a$ and $b$ are believed to be uniformly distributed, then buyer's order quantity $Q$ will be smaller, but the multiplier factor $\gamma$ will be larger, since the length of credit period $t$ offered by the seller will be shorter when compare with exponential and normal distributions. The buyer will gain more when seller's type follows the exponential distribution, whereas the seller will gain more when buyer believes that seller's type follows a normal

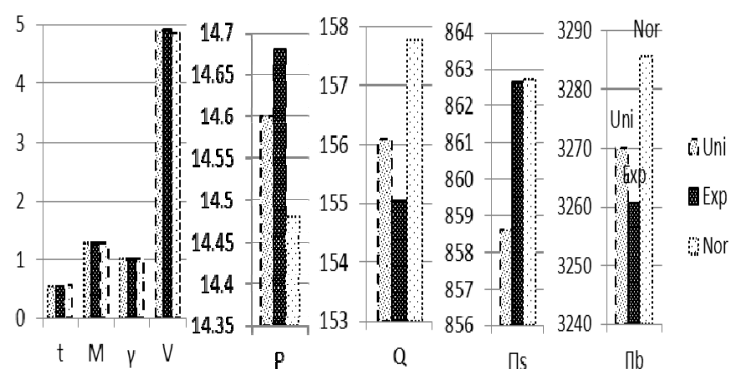

Figure 6. First scenario regarding to choice of probability density function. Uni: uniform, Exp: exponential, Nor: normal.

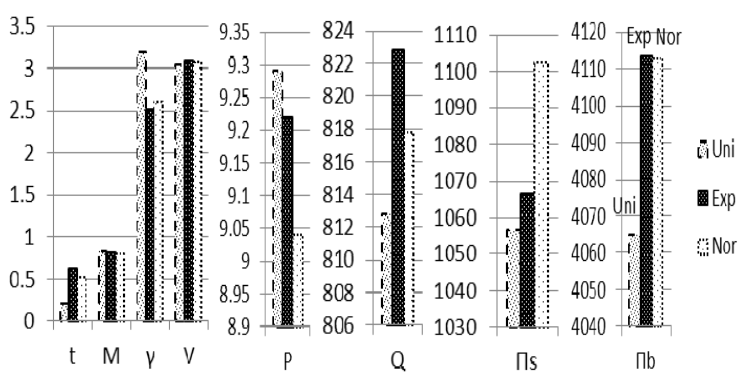

Figure 7. Second scenario regarding to choice of probability density function. Uni: uniform, Exp: exponential, Nor: normal.

distribution.

Figures 6 and 7 highlight how both seller and buyer's decisions and profits differ according to their choice of PDFs regarding each others' types.

\section{CONCLUSION}

In this paper, we presented two scenarios of sellerbuyer supply chain under both asymmetric and symmetric information structure. The interactions between the players are modelled as a two-person Stackelberg game. In the first scenario, the seller offers a credit option to the buyer and determines the length of credit period based on the buyer's best strategies; in the second scenario, based on the pre-existing credit period offered by the seller, the buyer imposes a multiplier factor extending the length of the credit period and compensate for this by offering to share his inventory cost. The numerical examples showed that in both scenarios, both seller and buyer's profits obtained from symmetric game are larger than those obtained from asymmetric information game. Furthermore, both buyer and seller's profits in the second scenario are larger than those obtained from the first scenario. We also observed that under asymmetric information, solutions for both seller and buyer are sensitive to player's belief over other player's type.

The current work can be extended to situations where there are multiple players in the supply chain, for instance, one seller offering different credit periods to 
multiple buyers with different demand functions. Finally, the research may be further extended to look at the consequences of the effects different kinds of information asymmetries have on the decisions each player brings to bear on the decisions they make.

\section{REFERENCES}

Abad, P. L. and Jaggi, C. K. (2003), A joint approach for setting unit price and the length of the credit period for a seller when end demand is price sensitive, International Journal of Production Economics, 83(2), 115-122.

Aggarwal, S. P. and Jaggi, C. K. (1995), Ordering policies of deteriorating items under permissible delay in payments, Journal of the Operational Research Society, 46(5), 658-662.

Chen, L. H. and Kang, F. S. (2010), Integrated inventory models considering the two-level trade credit policy and a price-negotiation scheme, European Journal of Operational Research, 205(1), 47-58.

Chung, K. H. (1998), A theorem on the determination of economic order quantity under conditions of permissible delay in payments, Computer and Operations Research, 25(1), 49-52.

Esmaeili, M. and Zeephongsekul, P. (2010), Seller-Buyer models of supply chain management with an asymmetric information structure, International Journal of Production Economics, 123(1), 146-154.

Esmaeili, M., Aryanezhad, M. B., and Zeephongsekul, P. (2009), A game theory approach in seller-buyer supply chain, European Journal of Operational Research, 195(2), 442-448.

Goyal, S. K. (1985), Economic order quantity under conditions of permissible delay in payment, Journal of the Operational Research Society, 36(4), 335-338.

Haley, C. W. and Higgins, R. C. (1973), Inventory policy and trade credit financing. Management Science, 20(4), 464-471.

Harsanyi, J. C. (1967), Games with incomplete information played by "Bayesian" players. Part I. The basic model, Management Science, 14(3), 159-182.

Harsanyi, J. C. (1968a), Games with incomplete information played by "Bayesian" players. Part II. Bayesian equilibrium points, Management Science, 14(5), 320-334.
Harsanyi, J. C. (1968b), Games with incomplete information played by "Bayesian" players. Part III. The basic probability distribution of the game, Management Science, 14(7), 486-502.

Jaber, M. Y. and Osman, I. H. (2006), Coordinating a twolevel supply chain with delay in payments and profit sharing, Computers and Industrial Engineering, 50(4), 385-400.

Jamal, A. M., Sarkar, B., and Wang, S. (2000), Optimal payment time for a retailer under permitted delay of payment to the wholesaler, International Journal of Production Economics, 66(1), 59-66.

Kim, J., Hwang, H., and Shinn, S. (1995), An optimal credit policy to increase supplier's profits with pricedependent demand functions, Production Planning and Control, 6(1), 45-50.

Lee, J. W. and Kim, D. (1993), Optimal and heuristic decision strategies for integrated product and marketing planning, Decision Sciences, 24(6), 1203-1214.

Ouyang, L. Y., Ho, C. H., and Su, C. H. (2009), An optimization approach for joint pricing and ordering problem in an integrated inventory system with order-size dependent trade credit, Computers \& Industrial Engineering, 57(3), 920-930.

Rasmusen, E. (2007), Game and Information: An Introduction to Game Theory (4th ed.), Blackwell Publishing Ltd., Malden, MA.

Sethuraman, R. and Tellis, G. (1991), An analysis of the tradeoff between advertising and price discounting, Journal of Marketing Research, 28(2), 160-174.

Shinn, S. W. (1997), Determining optimal retail price and lot size under day-terms supplier credit, Computer \& Industrial Engineering, 33(3-4), 717-720.

Thangam, A. and Uthayakumar, R. (2009), Two-echelon trade credit financing for perishable items in a supply chain when demand depends on both selling price and credit period, Computers \& Industrial Engineering, 57(3), 773-786.

Zhong, Y. G. and Zhou, Y. W. (2012), The model and algorithm for determining optimal ordering/tradecredit policy of supply chains, Applied Mathematics and Computation, 219(8), 3809-3825.

Zhou, Y. W., Zhong, Y. G., and Li, J. (2012), An uncooperative order model for items with trade credit, inventory dependent demand and limited displayedshelf space, European Journal of Operational Research, 223(1), 76-85. 


\section{APPENDIX}

\section{A Generalized Expression for the Buyer's Model}

In this appendix, we will show that the buyer's profit functions have the same mathematical expression in all the three cases displayed in Figure 1. For case 1, the buyer's profit function includes the following terms:

Sales revenue per cycle: $P D T$;

Purchase cost per cycle: $V D T$;

Marketing expenditure cost: $M D T$;

Ordering cost per cycle: $A_{b}$;

Storage cost excluding financing cost per cycle: $0: 5 I_{b} V D T^{2}$;

Capital gain on $[0, \gamma t]: 0: 5 I_{p} V D(\gamma t)^{2}$;

Financing inventory cost on $[\gamma t, T]: 0: 5 I_{c} V D(T-\gamma t)^{2}$;

Inventory financing cost for extended creditperiod: $I_{x} V D(\gamma t-t)^{2}$.

Using the assumption $I_{c}=I_{p}$, the sum of capital gain, storage cost and financing inventory cost is equal to

$$
0.5 I_{p} V D(\gamma t)^{2}-0.5 I_{b} V D T^{2}-0.5 I_{c} V D(T-\gamma t)^{2}=I_{c} V D T \gamma t-0.5 I_{b} V D T^{2}-0.5 I_{c} V D T^{2}
$$

Using the assumption $T=Q / D$, the buyer's annual profit function is:

$$
\Pi_{b}(P, M, Q, \gamma)=P D-V D-M D-A_{b} D Q^{-1}+I_{c} V D \gamma t-0.5 I_{b} V Q-0.5 I_{c} V Q-I_{x} V D(\gamma t-t)
$$

For cases 2 and 3, the first five terms and the last term in buyer's profit function are the same as in case 1, and the financing inventory cost is not present. Also, the capital gain on $[0, \gamma t]$ is:

$$
0.5 I_{p} V D T^{2}+I_{p} V D T(\gamma t-T)=I_{p} V D T \gamma t-0.5 I_{c} V D T^{2}
$$

So the buyer's annual profit function is:

$$
\Pi_{b}(P, M, Q, \gamma)=P D-V D-M D-A_{b} D Q^{-1}+I_{p} V D \gamma t-0.5 I_{b} V Q-0.5 I_{c} V Q-I_{x} V D(\gamma t-t)
$$

Since $I_{c}=I_{p}$, it follows that Eqs. (27) and (28) are equal. Therefore, the buyer's profit functions have the same expression in all three cases. $\square$ 